\title{
Megakaryocyte and Megakaryoblast Morphology Assessment
}

National Cancer Institute

\section{Source}

National Cancer Institute. Megakaryocyte and Megakaryoblast Morphology

Assessment. NCl Thesaurus. Code C135432.

An examination or assessment of the form and structure of megakaryocytes and megakaryoblasts present in a sample. 